\title{
Past and future uses of raw diffraction data
}

George Phillips $^{1}$

${ }^{1}$ BioSciences/Chemistry Rice Univeristy, Houston, United States

E-mail: georgep@rice.edu

For over 100 years, X-ray crystallography has been provided information on atomic and molecular structure. With data first recorded on photographic plates, then films and later in digital form, the wealth of data has led to Nobel prize winning work in many fields of science. The ethics of sharing data crystallographic sharing likely go back 100 years as well, with the well discussed use or misuse of Rosalind Franklin's famous photo 51 during the discovery of the double helix of DNA. Recent studies on the reproducibility of scientific works also contribute to the dialog on appropriate sharing and use of original data.

To the credit of the field of crystallography, the sharing of the diffraction data has (slowly) but steadily improved, with many journal editors (but not all) requiring deposition of the atomic coordinates and sometimes the processed list of diffraction intensities as a condition of publication. The Protein Data Bank (PDB) has served the community well in this regard, accepting over one hundred thousand individual submissions. More recently, the question of whether to archive for the public the original digital images has been discussed with several organizations offering to collect, minimally annotate, and distribute these 'raw' diffraction images. The PDB does not currently offer this service, probably due to lack of resources for the labor required for curation, as electronic storage is relatively inexpensive. New time-resolved diffraction experiments and non-Bragg scattering analysis give reasons to evaluate the value of shared raw data.

Presented in this talk are some use cases for why it might be interesting to have such original data archived, even if much of it is never reused.

Supported by NSF grants for BioXFEL Science and Technology Center \#1231306 and NIH grants GM109456 and GM098248. Keywords: Diffraction images, data sharing, data processing 